\title{
Frequent PIK3CA mutations in eutopic endometrium of patients with ovarian clear cell carcinoma
}

\author{
Kosuke Murakami ${ }^{1}{ }^{1}$, Akiko Kanto ${ }^{1}$, Kazuko Sakai ${ }^{2}$, Chiho Miyagawa ${ }^{1}$, Hisamitsu Takaya ${ }^{1}$, Hidekatsu Nakai ${ }^{1}$, Yasushi Kotani ${ }^{1}$, \\ Kazuto Nishio (iD) ${ }^{2}$ and Noriomi Matsumura (iD) ${ }^{1 凶}$
}

(c) The Author(s) 2021

Recent studies have reported cancer-associated mutations in normal endometrium. Mutations in eutopic endometrium may lead to endometriosis and endometriosis-associated ovarian cancer. We investigated PIK3CA mutations (PIK3CAm) for three hotspots (E542K, E545K, H1047R) in eutopic endometrium in patients with ovarian cancer and endometriosis from formalin-fixed paraffinembedded specimens by laser-capture microdissection and droplet digital PCR. The presence of PIK3CAm in eutopic endometrial glands with mutant allele frequency $\geq 15 \%$ were as follows: ovarian clear cell carcinoma (OCCC) with PIK3CAm in tumors, 20/300 hotspots in 11/14 cases; OCCC without PIK3CAm, 42/78 hotspots in 11/12 cases; high-grade serous ovarian carcinoma, 8/45 hotspots in 3/5 cases; and endometriotic cysts, $5 / 63$ hotspots in $5 / 6$ cases. These rates were more frequent than in noncancer nonendometriosis controls (7/309 hotspots in 5/17 cases). In OCCC without PIK3CAm, 7/12 (58\%) cases showed multiple hotspot mutations in the same eutopic endometrial glands. In 3/54 (5.6\%) cases, PIK3CAm was found in eutopic endometrial stroma. Multisampling of the OCCC tumors with PIK3CAm showed intratumor heterogeneity in three of eight cases. In two cases, PIK3CAm was detected in the stromal component of the tumor. Homogenous PIK3CAm in the epithelial component of the tumor matched the mutation in eutopic endometrial glands in only one case. Eutopic endometrial glands in ovarian cancer and endometriosis show high frequency of PIK3CAm that is not consistent with tumors, and multiple hotspot mutations are often found in the same glands. While the mutations identified in eutopic endometrium may not be driver mutations in the patient's cancer, these are still driver mutations but this specific clone has not undergone the requisite steps for the development of cancer.

Modern Pathology (2021) 34:2071-2079; https://doi.org/10.1038/s41379-021-00861-3

\section{INTRODUCTION}

Ovarian cancer is a gynecologic malignancy with one of the worst prognoses. Ovarian clear cell carcinoma (OCCC) occurs frequently in Japan and accounts for approximately one quarter of all ovarian cancer cases [1]. OCCC is an endometriosis-associated ovarian cancer (EAOC) [2] and was previously thought to arise from endometriotic cysts (ECs) [3]. However, later studies have not confirmed this possibility, and the origin of OCCC remains unclear.

Recent advances in sequencing technology have revealed the presence of numerous cancer-associated mutations in the eutopic endometrial epithelium in healthy patients [4, 5]. Cancerassociated mutations, including mutations in PIK3CA, are frequently found in deep infiltrating endometriosis (DIE) [6]. Because the same mutations in eutopic endometrium are also found in $E C s$, endometriosis is thought to be caused by the reflux of eutopic endometrium with gene mutations [7]. Notably, a case of clonal lineage from eutopic endometrium to endometriosis and OCCC was reported [8]. Therefore, eutopic endometrium has recently attracted a great deal of interest as a potential origin of EAOC [9]. A previous study showed that endometrial glands are composed of monoclonal cell populations [10]. Therefore, to clarify whether OCCC arises from eutopic endometrial cells, it is necessary to perform mutation analysis for individual endometrial glands and compare the results with mutations in ovarian tumors.

Droplet digital PCR (ddPCR) is a technology that amplifies even a very small amount of DNA to enable mutation analysis [11]. In a recent study in endosalpingiosis, the authors collected formalinfixed paraffin-embedded (FFPE) samples from glands by lasercapture microdissection (LCM), and ddPCR was used to analyze hotspot mutations in BRAF and KRAS [12]. We hypothesized that this technique would allow for mutation analysis of individual endometrial glands from FFPE samples of the uterus.

Specific mutations in PIK3CA have been shown to activate the $\mathrm{PI}$ IK/Akt/mTOR pathway and are deeply involved in human carcinogenesis [13]. PIK3CA is one of the most frequently mutated genes along with ARID1A in OCCC [14], and co-mutation of Pik3ca and Arid $1 a$ in mouse ovaries causes cancer similar to human OCCC [15]. In human cancers, including OCCC, three hotspot mutations in PIK3CA have been identified: E542K, E545K, and H1047R $[13,16,17]$.

In this study, we examined PIK3CA hotspot mutations in eutopic endometrium in ovarian cancer, especially OCCC, and endometriosis cases by ddPCR and compared them with mutations in tumors. This study is the first report to compare mutations

${ }^{1}$ Department of Obstetrics and Gynecology, Kindai University Faculty of Medicine, Osaka, Japan. ${ }^{2}$ Department of Genome Biology, Kindai University Faculty of Medicine, Osaka, Japan. ${ }^{凶}$ email: noriomi@med.kindai.ac.jp 
between tumors and normal eutopic endometrium in multiple ovarian cancer cases.

\section{MATERIALS AND METHODS \\ Sample collection}

Patients who underwent surgery at the Department of Obstetrics and Gynecology, Kindai University Hospital, between January 1999 and December 2019 were included in this study. For OCCC cases, FFPE samples of tumors of the ovary, eutopic endometrium, and coexisting endometriosis were obtained. We also obtained FFPE samples of tumors of the ovary and eutopic endometrium for high-grade serous ovarian carcinoma (HGSOC) and EC cases. As controls, FFPE samples of eutopic endometrium were obtained from patients whose uterus had been removed due to benign disease or cervical intraepithelial neoplasia without endometriosis or adenomyosis. Pathological diagnosis of each sample was made by two pathologists.

\section{DNA extraction}

For OCCC and HGSOC tumors, after confirming the tumor area by hematoxylin and eosin staining, FFPE samples were sliced at a thickness of $3 \mu \mathrm{m}$ and macrodissection was performed using a razor to obtain only the tumor area. The collected samples were deparaffinized, and DNA was extracted using the QIAamp DNA Micro Kit (Qiagen, Hilden, Germany) following the manufacturer's protocol. In six cases of OCCC, five tumor sections that were cut at least $1 \mathrm{~cm}$ apart were sampled with macrodissection. In seven cases of OCCC, five areas of the tumor epithelial component and three areas of the tumor stromal component were sampled with LCM (described below) from one section. In endometriosis and eutopic endometrium, hematoxylin and eosin staining was used to confirm the presence of endometriotic epithelium or endometrial glandular epithelium. FFPE samples were sliced to a thickness of $10 \mu \mathrm{m}$ and placed on a glass slide with foil (Leica, Wetzlar, Germany), stained with toluidine blue (Supplementary Table S1), and cut out by LCM using a Leica LMD7000 (Leica, Wetzlar, Germany) (Fig. 1A-E). For each case, 4-12 eutopic endometrial glandular epithelium samples were sampled individually, and 3-4 endometrial stroma in close proximity were grouped together to form one sample. DNA was extracted from the collected samples using the QIAamp DNA Micro Kit (Qiagen, Hilden, Germany) according to the manufacturer's protocol.

\section{ddPCR assays}

Reactions were prepared using $1.1 \mu \mathrm{L}$ of PrimePCR for ddPCR PIK3CA E542K, E545K, or H1047R (Bio-Rad Laboratories, Hercules, CA, USA), $11 \mu \mathrm{L}$ of ddPCR supermix for probes (no dUTP) (Bio-Rad Laboratories), $5.9 \mu \mathrm{L}$ of distilled water, and $4 \mu \mathrm{L}$ of extracted DNA for a total of $22 \mu \mathrm{L}$. Droplets were prepared using the Automated Droplet Generator (Bio-Rad Laboratories). After amplification in a thermal cycler (Supplementary Table S2), the numbers of wild-type and mutant copies per $20 \mu \mathrm{L}$ were counted using the QX200 Droplet Digital PCR System (Bio-Rad Laboratories). The mutant allele frequency (MAF) was defined as the ratio of the number of mutant copies to the total number of copies for each hotspot. If any one of the three hotspots (PIK3CA E542K, E545K, or H1047R) was not detected in either wild-type or mutant copies, that sample was excluded from the analysis.

The cutoff of MAF was set at $15 \%$ based on the previous report [7]. Samples with a MAF $\geq 15 \%$ of PIK3CA are shown in Fig. 2A. The heat map of MAF without setting the cutoff value is shown in Fig. $3 C$ and Supplementary Fig. S1.

\section{Analysis of public data}

A previous report examined endometrial cancer and ovarian cancer cases using Pap smear and plasma, and we downloaded the supplemental target sequencing data for each available specimen [18]. We selected ovarian cancer cases in which PIK3CA mutation was detected in the tumor, Pap smear, or plasma and examined the relationship between mutations.

\section{Statistical analysis}

Statistical analyses were performed using the GraphPad Prism ver. 9.0.0 (GraphPad Software, San Diego, CA, USA). Fisher's exact test was used to compare the proportions between groups, and Mann-Whitney test or
Kruskal-Wallis test was used to compare the MAF. The Kaplan-Meier method was used to calculate overall survival, and the log-rank test was used to compare the curves. The correlation between age and MAF was analyzed by Spearman's rank correlation analysis, and $P$ values less than 0.05 were considered statistically significant.

\section{RESULTS \\ PIK3CA mutations in ovarian cancer and endometriotic epithelium}

We initially obtained FFPE samples for OCCC tumors from 60 cases. The mean age of the patients was $53.9 \pm 10.0$ years, with $70 \%$ of patients in FIGO stage 1 or 2 and $30 \%$ in stage 3 or 4 . When the cutoff value of MAF was set at $15 \%, 15$ of the 60 tumors (25\%) had PIK3CA E542K, E545K, or H1047R mutation. In addition to these cases, there was one case with a MAF of 5.5\% (PIK3CA E542K mutation). In these 16 cases, PIK3CA E542K, E545K, and H1047R mutations in the tumor were mutually exclusive. We divided the 60 patients into two groups according to the presence of PIK3CA mutation in the tumor and found no significant difference in overall survival between the two groups (Supplementary Fig. S2A). There was also no significant difference in age between the two groups (Supplementary Fig. S2B).

Among the 16 cases of OCCC with PIK3CA mutation in the tumor, 14 cases had analyzable eutopic endometrium. In three of these cases, four FFPE samples containing EC or DIE not adjacent to the tumor were available. All three cases showed PIK3CA mutation in the endometriotic epithelium. Two of the cases showed E545K mutation in the endometriotic epithelium with H1047R mutation in the tumor (Fig. 2A; Supplementary Fig. S1; cases $\mathrm{a}$ and $\mathrm{b}$ ). In the other case, E545K and E542K mutations occurred simultaneously in the endometriotic epithelium with E545K mutation in the tumor (Fig. 2A; Supplementary Fig. S1; case e).

For comparison with the 14 OCCC cases with PIK3CA mutation in the tumor, we obtained FFPE samples of tumor and eutopic endometrium from 12 OCCC cases without PIK3CA mutation in the tumor, $5 \mathrm{HGSOC}$ cases that were unrelated to endometriosis, and 6 EC cases. In addition, FFPE samples of control endometrium from 17 healthy controls were obtained. The characteristics of the OCCC, HGSOC, EC, and control groups are shown in Table 1. There was no significant difference in age between the groups (Fig. 2B). There were no cases of $\mathrm{HGSOC}$ with PIK3CA mutation with MAF $\geq$ $1 \%$ in the tumor (Fig. 2A; Supplementary Fig. S1). Endometriotic epithelium of two of the six EC cases showed simultaneous MAF $\geq$ $15 \%$ mutation in E542K and E545K (Fig. 2A).

\section{PIK3CA mutations in eutopic endometrial glandular epithelium and stroma and comparisons with tumors} In the overall 54 cases (OCCC, HGSOC, EC, and controls), ddPCR was performed on 374 endometrial glands. A total of 265 endometrial glands $(71 \%)$ for which MAF could be calculated at all three hotspots were included in the analysis. The number of cases with endometrial glands with MAF $\geq 15 \%$ in E542K, E545K, or H1047R was significantly higher in the OCCC with PIK3CA mutation group and the OCCC without PIK3CA mutation group compared with the control group (Table 2). The number of hotspots with $\mathrm{MAF} \geq 15 \%$ was significantly higher in the OCCC with PIK3CA mutation group, the OCCC without PIK3CA mutation group, the HGSOC group, and the EC group compared with the control group (Table 2). In comparing the distribution of MAF of all endometrial glands within each group, the MAF was significantly higher in the OCCC with PIK3CA mutation group, the OCCC without PIK3CA mutation group, the HGSOC group, and the EC group compared with the control group (Table 2). Interestingly, there were some cases with two or three hotspots of MAF $\geq 15 \%$ mutations among the three hotspots in the same gland, which 

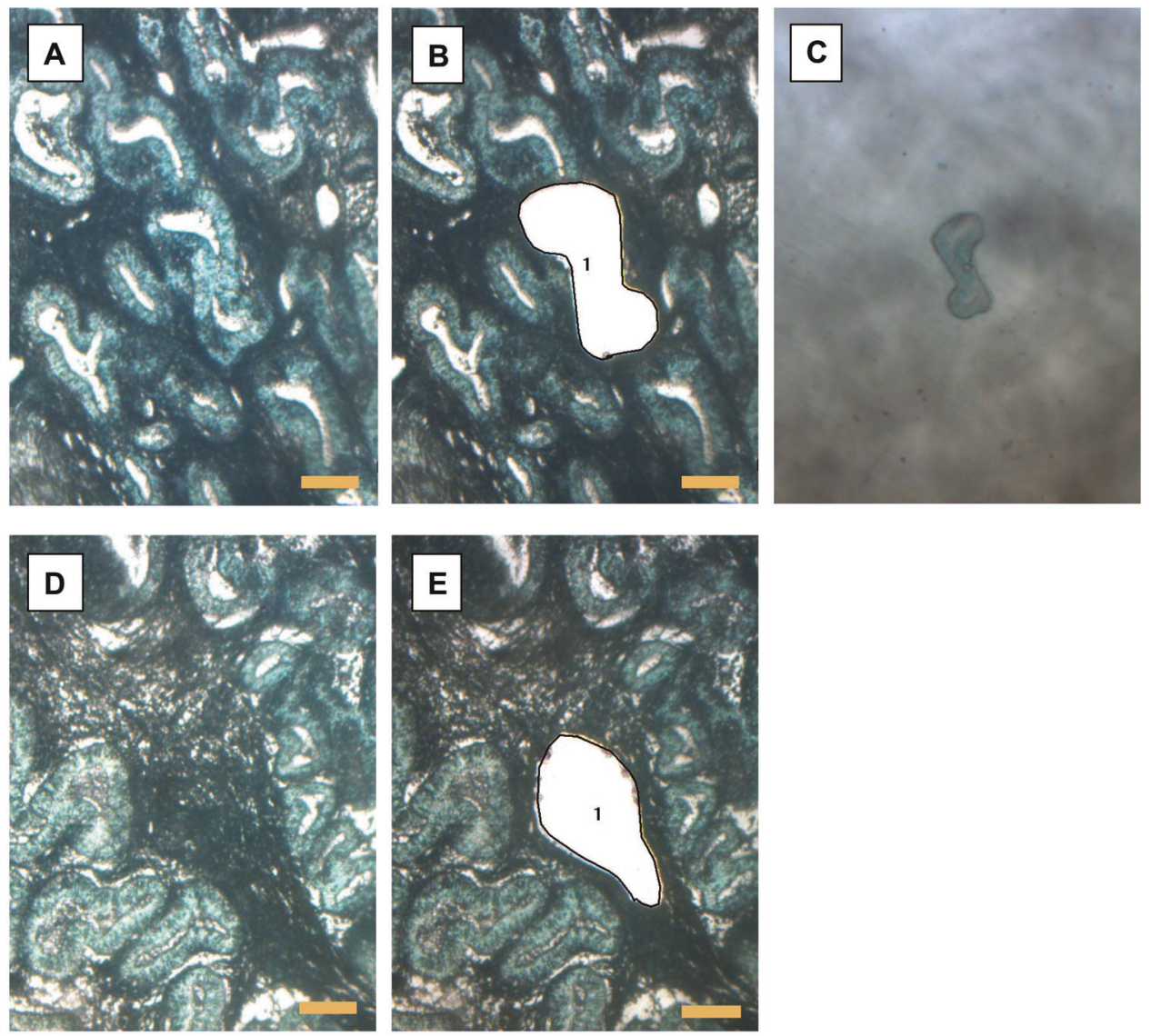

Fig. 1 Examples of laser-capture microdissection. A, B Endometrial glands of secretory phase (magnification, 63×). C Collected endometrial glands. D, E Endometrial stroma of secretory phase (63x). Scale bars: $100 \mu \mathrm{m}$.

were particularly common in the OCCC without PIK3CA mutation group (Table 2). There was no correlation between age and MAF of PIK3CA mutation in endometrial glands (Supplementary Fig. S3). The MAF of PIK3CA mutation in endometrial glands did neither correlate with endometrial status (proliferative phase, secretory phase, or atrophy) nor did it correlate with FIGO stage in ovarian cancer cases (data not shown).

We sampled eutopic endometrial stroma from all 54 cases by LCM and PIK3CA mutation analysis was performed. Endometrial stroma with $\mathrm{MAF} \geq 15 \%$ was detected in only three cases $(5.6 \%)$, three out of 162 hotspots (1.9\%). These three cases included two OCCC cases and one control case (Fig. 2A).

Among the 14 cases of OCCC with PIK3CA mutation, only three cases (21.4\%) had PIK3CA mutation with MAF $\geq 15 \%$ in the eutopic endometrial glandular epithelium in a single hotspot coincident with the tumor (Fig. 2A; cases b, g, and h). Conversely, the two OCCC with PIK3CA mutation cases showed coincident sites of PIK3CA mutations in the tumor and endometrial stroma; one of which had H1047R mutation in the endometrial stroma with H1047R mutation in the tumor and the other had E545K mutation in the endometrial stroma with E545K mutation in the tumor (cases $\mathrm{c}$ and $\mathrm{f}$ ).

\section{Comparison of PIK3CA mutation in eutopic endometrium and tumor, and analysis of intratumor heterogeneity}

Analysis of the intratumor heterogeneity of PIK3CA mutations may help provide insights into the origin of OCCC. For example, if eutopic endometrial cells with PIK3CA mutation as an oncogenic driver are the origin of OCCC, then all tumor cells of OCCC would have the PIK3CA mutation (Fig. 3A). Therefore, in six cases of
OCCC, additional macrodissection samplings were performed from four tumor sections more than $1 \mathrm{~cm}$ apart from each other. In addition, in seven OCCC cases, samples were collected by LCM from five epithelial components and three stromal components of tumors in very close proximity to each other within the same sample as the original macrodissection (Fig. 3B). The additional macrodissection showed that in three of the four OCCC cases with PIK3CA mutation in the tumor, the additional sample had the same hotspot mutation as the original sample (Fig. 3C; cases a, b, and d). However, in one case, the additional sample did not have the mutation (Fig. 3C; case e). The LCM analysis showed that in some cases, all epithelial components had the same hotspot mutation as the original tumor part (Fig. 3C; cases $\mathrm{c}, \mathrm{f}$, and $\mathrm{h}$ ), while in others, some epithelial components did not have PIK3CA mutation (Fig. 3C; cases $b$ and g). In the majority of the LCM samples, the MAF was higher than in the macrodissection samples (Fig. 3C). Among OCCC cases with PIK3CA mutation in the tumor, only one case had homogeneous PIK3CA mutation in the epithelial component of the tumor and a single hotspot mutation with $M A F \geq 15 \%$, consistent with the tumor, in the eutopic endometrial glandular epithelium (Fig. 3C; case h). Conversely, in two cases with PIK3CA mutations with MAF $\geq 15 \%$ in a single hotspot in the eutopic endometrial stroma consistent with the tumor, the PIK3CA mutations in the epithelial component of the tumor were homogeneous (Fig. 3C; cases $\mathrm{c}$ and f). In two cases, the stromal component of the tumor showed the same hotspot mutation as the epithelial component of the tumor (Fig. 3C; cases $f$ and g). In OCCC cases without PIK3CA mutation in the original sample of the tumor, neither the additional macrodissection sample nor the LCM sample had PIK3CA mutation (Fig. 3C; cases i-k). 
A

OccC

HGSOC

EC

Control

$\mathrm{n}=26$

$\mathrm{n}=5$

$\mathrm{n}=6$

$\mathrm{n}=17$

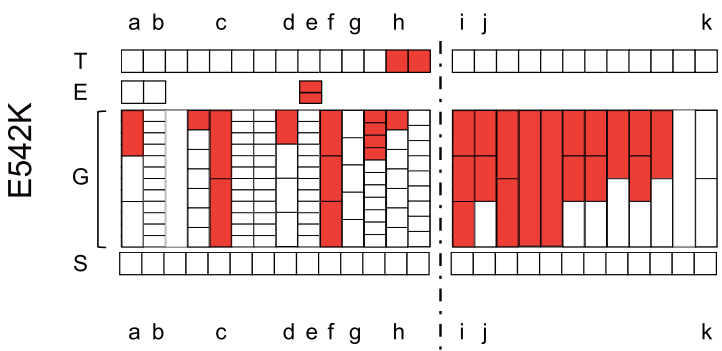

k
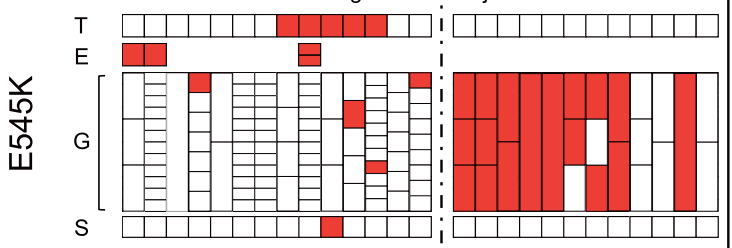

k
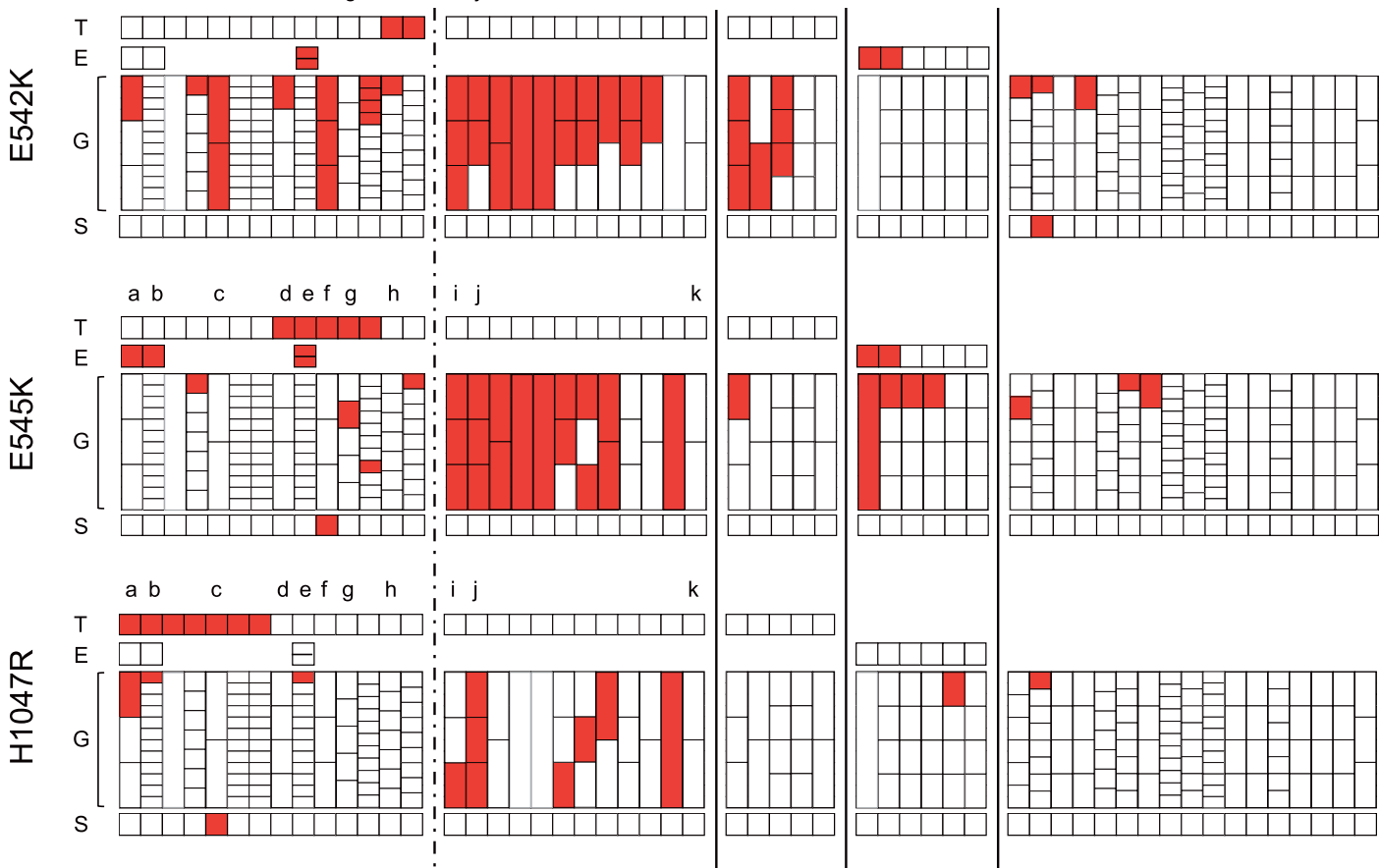

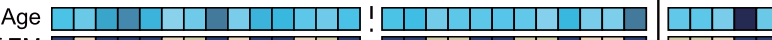
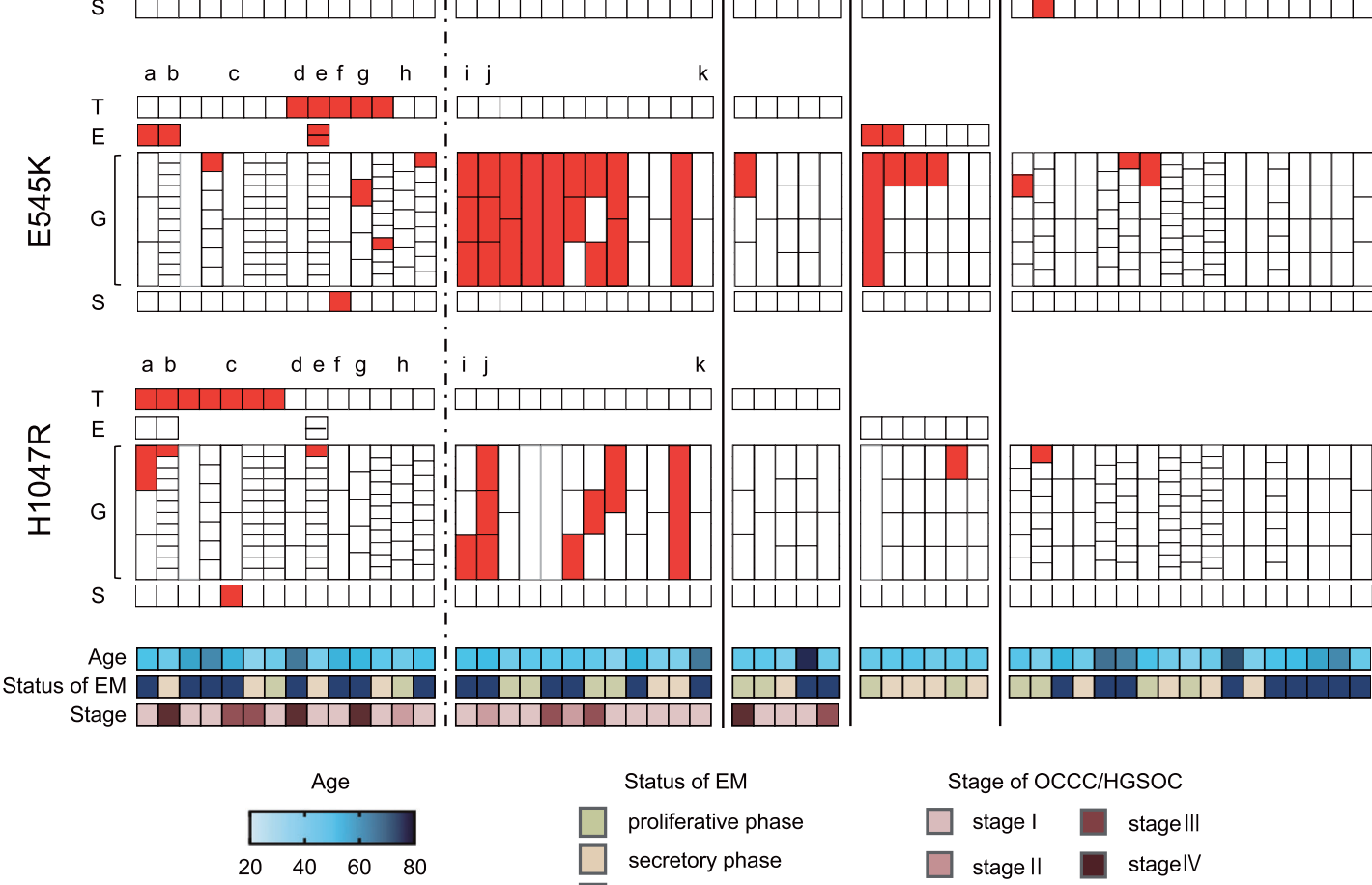

Status of EM

$\square$ proliferative phase

atrophy
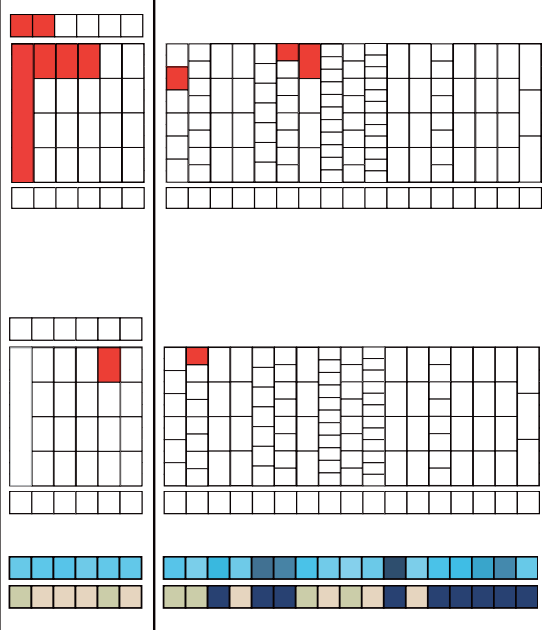

k \begin{tabular}{l|l|l|l|}
\hline & & 1 & \\
\hline
\end{tabular}
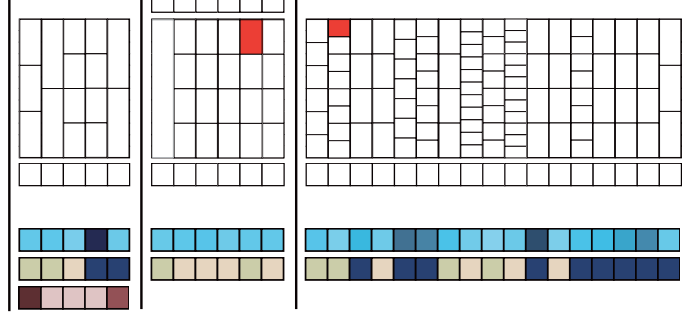

B

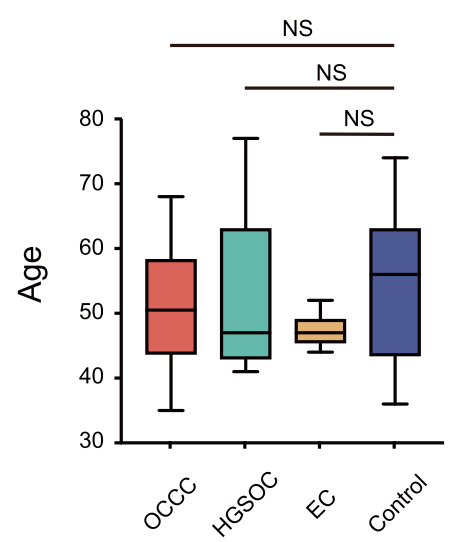

Fig. 2 PIK3CA mutations in OCCC, HGSOC, EC, endometrial glands, and endometrial stroma. A PIK3CA mutation in OCCC, HGSOC, EC, endometrial glands, and endometrial stroma in all cases. Tumor, endometriosis, endometrial glands, and endometrial stroma of each case were divided into three hotspots and arranged by sample. The spots with mutant allele frequency $\geq 15 \%$ are shown in red. For the OCCC cases, the cases on the left side of the dotted line have PIK3CA mutation in the tumor, while the cases on the right side are cases without PIK3CA mutation. a-k show cases which tumors were macro- or microdissected and multisampled (details are shown in Fig. 3C). B Comparison of the patient age of each group. The box and whisker plot shows the age of each group. OCCC ovarian clear cell carcinoma, HGSOC highgrade serous ovarian carcinoma, EC endometriotic cyst, $\mathrm{T}$ tumor, $\mathrm{E}$ endometriosis, $\mathrm{G}$ endometrial gland, $\mathrm{S}$ endometrial stroma, EM endometrium, NS not significant. 
A

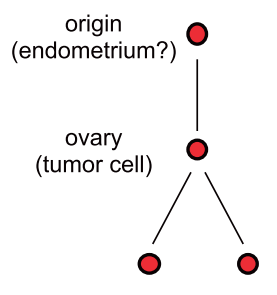

heterogeneity

$(-)$

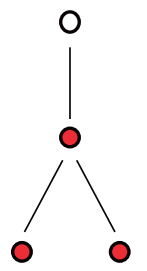

$(-)$

B
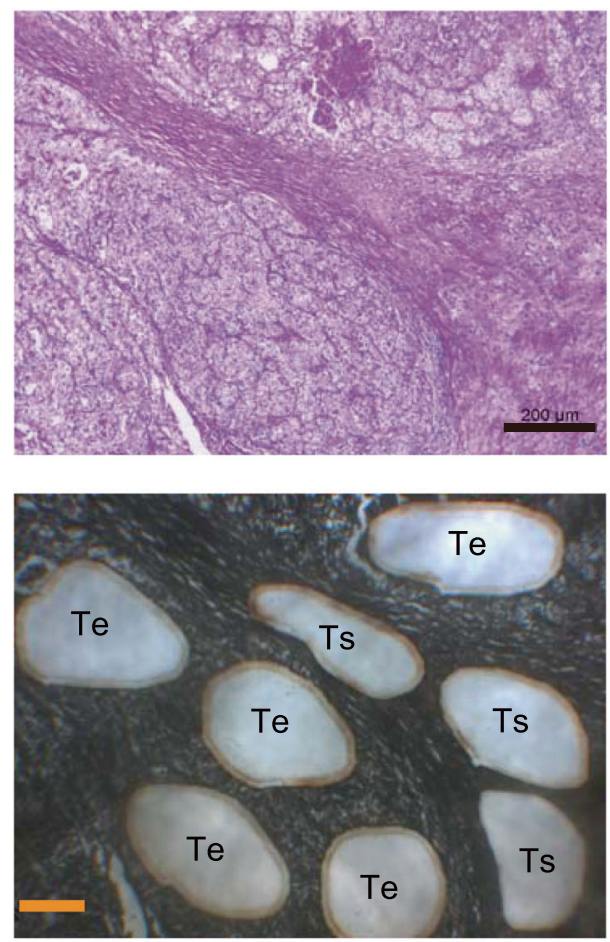

C

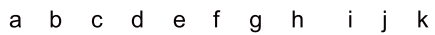
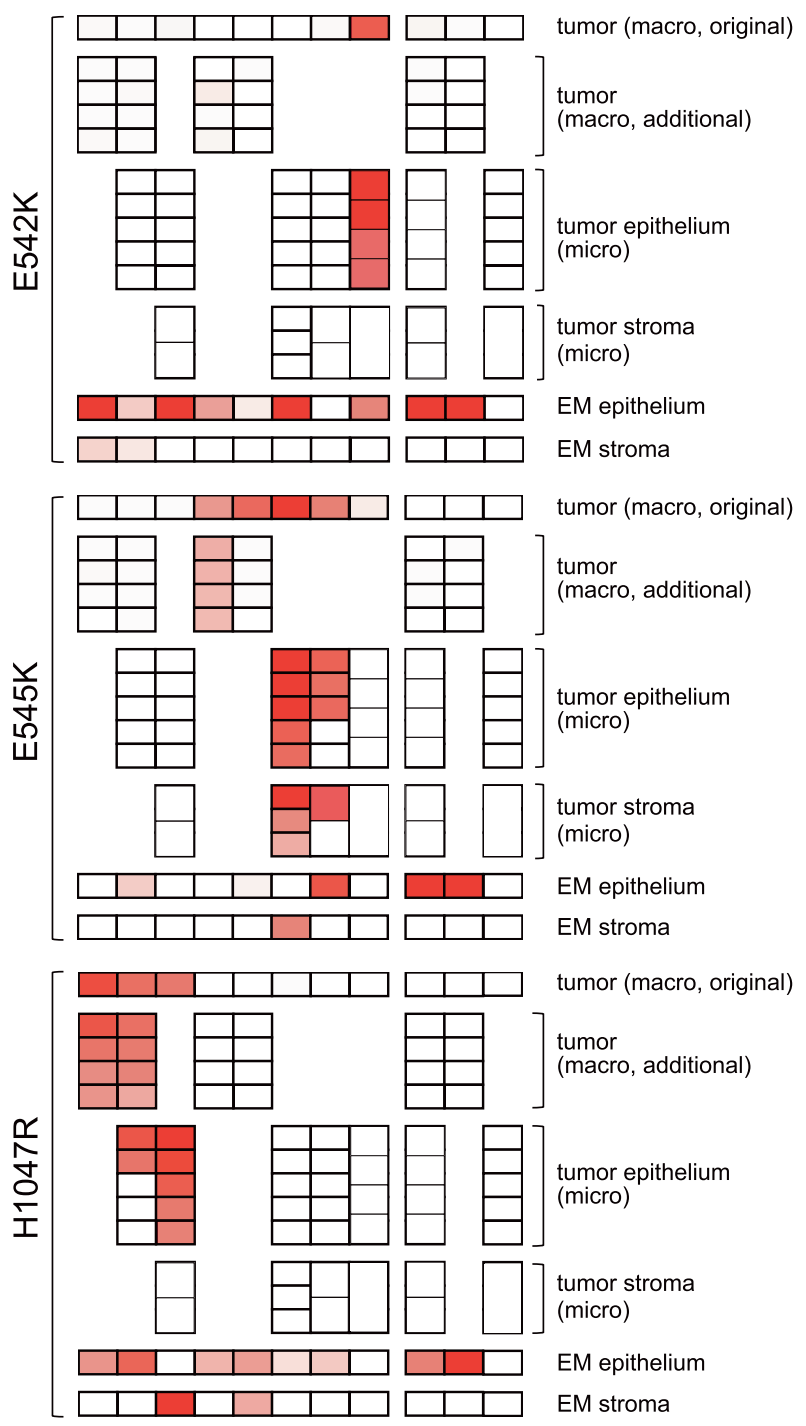

MAF (\%)

$0 \quad 102030 \quad 4050$

Fig. 3 PIK3CA mutations in tumors by macro- and microdissection. A Association between PIK3CA mutations in tumor cells of origin and intratumor heterogeneity. If cells with driver PIK3CA mutations arise outside of the ovary, such as in the endometrium, and are transported to the ovary to form a tumor (left) or if the tumor cells have driver PIK3CA mutations at the time they arise in the ovary (middle), then PIK3CA mutations are likely to be present in all tumor cells. However, if PIK3CA mutations are not present at the time the tumor cells arise and emerge later, intratumor heterogeneity of PIK3CA mutations would occur (right). B Example of laser-capture microdissection. Clear cell carcinoma (case $\mathrm{g}$ from Fig. 2, hematoxylin and eosin staining; magnification 50x, toluidine blue staining; magnification $63 \times$ ). "Te" represents the epithelial component of the tumor and "Ts" represents the stromal component of the tumor. Scale bar: hematoxylin and eosin staining; 200um, toluidine blue staining; $100 \mu \mathrm{m}$. C MAF of the epithelial component and stromal component of the tumor and eutopic endometrial glandular epithelium and stroma. MAFs of PIK3CA by macro- and microdissection are shown in the heat map. For the endometrial glandular epithelium, the sample with the highest MAF was extracted. The cases a-k in C are the same as those shown in Fig. 2A. The tumor of cases a, b, $d$, e, i, and $j$ were multiple macrodissected. The epithelial and stromal components of the tumor of cases $b, c, f-i$, and $k$ were microdissected. Macro macrodissection, micro laser-capture microdissection, EM endometrium, MAF mutant allele frequency.

\section{Comparison of MAF in OCCC, endometriosis, and eutopic endometrium}

We compared the MAF of samples in which mutant alleles of PIK3CA were detected in ten groups: OCCC (epithelial and stromal components of tumors sampled by LCM), endometriosis (comorbid endometriosis in OCCC and ECs), and eutopic endometrium (endometrial glands of OCCC with PIK3CA mutation in the tumor, OCCC without PIK3CA mutation in the tumor, HGSOC, EC, healthy control, and endometrial stroma). Significant differences in MAF were observed among the groups (Kruskal-Wallis test, $P<0.001$ ). OCCC had a high MAF, and there were no samples with a MAF below $10 \%$ (Fig. 4). High MAF was observed in endometriosis associated with OCCC, but low MAF was observed in ECs unrelated to OCCC (Fig. 4). Eutopic endometrium showed a large number of samples with low MAF, except in the OCCC without PIK3CA mutation group (Fig. 4). 
Table 1. Characteristics of patients.

\begin{tabular}{|lllll|}
\hline & OCCC & HGSOC & EC & Control \\
\hline$n$ & 26 & 5 & 6 & 17 \\
\hline Age (mean) & $51.3 \pm 9.5$ & $51.8 \pm 14.0$ & $47.3 \pm 2.7$ & $53.4 \pm 11.0$ \\
\hline $\begin{array}{l}\text { Disease } \\
\quad \text { Stage I }\end{array}$ & 16 & 3 & - & - \\
\hline Stage II & 3 & 0 & - & - \\
\hline $\begin{array}{l}\text { Stage III } \\
\text { Stage IV }\end{array}$ & 4 & 1 & - & - \\
\hline $\begin{array}{l}\text { Cervical } \\
\text { intraepithelial } \\
\text { neoplasia }\end{array}$ & - & 1 & - & - \\
\hline $\begin{array}{l}\text { Benign ovarian } \\
\text { tumor }\end{array}$ & - & - & - & 14 \\
\hline $\begin{array}{l}\text { Status of endometrium } \\
\text { Proliferative phase }\end{array}$ & 6 & - & - & 3 \\
\hline $\begin{array}{l}\text { Secretory phase } \\
\text { Atrophy }\end{array}$ & 6 & 1 & & \\
\hline
\end{tabular}

OCCC ovarian clear cell carcinoma, HGSOC high-grade serous ovarian carcinoma, EC endometriotic cyst.

\section{Analysis of previously reported public data}

We searched for data on PIK3CA mutations in noncancerous sites in ovarian cancer from previous reports. Wang et al. performed genetic mutation analysis in ovarian cancer and endometrial cancer using Pap smear samples, Tao brush samples, and plasma sample-derived cell-free DNA [18]. The authors reported that cancer-associated mutations were found in ovarian cancer cases with a sensitivity of $63 \%$ and a specificity of $100 \%$. Therefore, we compared PIK3CA mutations in tumors and nontumor samples in the publicly available ovarian cancer data from the study. Since the proportion of cases in which Tao brush samples were obtained was very small, Pap smear samples and plasma samples were examined.

In Wang's cohort, 159 cases of HGSOC were included among 201 cases of ovarian cancer, and tumor PIK3CA mutations were found in only two cases (1.2\%). In ovarian cancer cases, 25 cases showed PIK3CA mutation in at least a tumor, Pap smear, or plasma sample. Only 6 of 25 (24\%) cases had the same PIK3CA mutation in the tumor and Pap smear or plasma (Supplementary Fig. S4). In ten cases in which no PIK3CA mutation was found in the tumor (eight HGSOC, one endometrioid carcinoma, and one mucinous carcinoma), PIK3CA mutation was detected only in the Pap smear or plasma sample (Supplementary Fig. S4).

\section{DISCUSSION}

This study was conducted to examine our hypothesis that EAOC originates from eutopic endometrium [9] and determine whether eutopic endometrium has a high frequency of mutations identical to EAOC. However, the results obtained were quite different from our predictions.

In this study, we focused on three hotspots of PIK3CA, E542K, E545K, and H1047R, which are frequent in OCCC, using FFPE samples used in clinical practice to ensure a sufficient number of cases. The frequency of OCCC cases with PIK3CA mutations was reported to be $30-50 \%[16,19-21]$. In this study, when the cutoff value of MAF was set at $15 \%$ based on the previous report [7], the frequency of OCCC cases with PIK3CA mutations was 25\%, which was slightly lower than the previously reported rate, but we consider this to be a reasonable frequency because only three hotspots were studied, and it may reflect the general OCCC population. The PIK3CA mutations in three hotspots in the tumor were mutually exclusive (Figs. $2 \mathrm{~A}$ and $3 \mathrm{C}$ ), which is a natural result as oncogenic driver mutation $[22,23]$.

In contrast to our findings in OCCC, eutopic endometrial glandular epithelium showed an unexpectedly high frequency of PIK3CA mutations. In a previous report that examined cancerassociated mutations in histologically normal endometrium by macrodissection, MAF was at most $10 \%$ [4]. Even in a report on cancer-associated mutations in DIE, MAF was at most 20\% [6]. Conversely, when endometrial glands are selectively sampled, MAF is often higher than $30 \%$ [5]. In our present study, there were many endometrial glands with $\mathrm{MAF}>30 \%$ (Fig. $2 \mathrm{~A}$ ), which is consistent with the report. The most impressive data we obtained in this study were the high frequency of eutopic endometrial glandular epithelium-specific PIK3CA mutations in OCCC cases without PIK3CA mutations in the tumor. Interestingly, mutations in two or three of the three hotspots were often observed in the same gland (Fig. 2A, Table 2). Recent reports have shown that cancer-associated mutations are frequently found in many normal tissues such as esophagus, skin, trachea, and colon, as well as the endometrium [24-29]. In esophageal cancer cases, the frequency of mutations in NOTCH1, a cancer-associated gene, was significantly higher in the normal epithelium of the esophagus adjacent to the cancer than in the esophageal cancer (66\% vs. $15 \%)$, and there were multiple mutations in NOTCH1 in the same clone of normal esophageal epithelium [24]. In addition, meticulous analysis of normal esophageal epithelium did not show a lineage to esophageal cancer. These results seem to indicate a similar phenomenon to our data. Furthermore, if PIK3CA mutations in eutopic endometrium are driver mutations of OCCC, there would be no heterogeneity of PIK3CA mutations in tumors (Fig. 3A). However, intratumor heterogeneity of PIK3CA mutations was observed in three of the eight cases in which multisampling of the tumor was performed, and only one case showed both PIK3CA mutation in the eutopic endometrial glandular epithelium and homogeneous PIK3CA mutation in the tumor (Fig. 3C). These results suggest that PIK3CA mutations in eutopic endometrial glandular epithelium may not be driver mutations in the genesis of OCCC.

Similar to the results in eutopic endometrium, PIK3CA mutations in the endometriotic epithelium coexisting with OCCC were not identical to those in the tumor. Previous studies reported that in EAOC, the same mutations found in cancer were also found in endometriotic lesions adjacent to the cancer $[19,30]$. However, in our study, we isolated and examined endometriotic epithelium at a site remote from the cancer by LCM, which is different from the method used in previous reports. In endometriotic epithelium, multiple hotspot mutations were also found in the same sample (Fig. 2A). While the mutations identified in the normal endometrium or endometriosis may not be drivers in the patient's cancer, these mutations are still driver mutations but this specific clone has not undergone the requisite steps for the development of cancer.

With regard to the frequency of gene mutations in normal tissues and cancer, previous studies reported that mutant fractions are higher in cancer tissues than in normal tissues [31]. Similar to our results, Suda et al. also showed that MAF was higher in OCCC than in eutopic endometrium [8]. In our analysis of LCM samples, the high MAF of PIK3CA in the tumor of OCCC and endometriosis associated with OCCC suggested clonal expansion in these samples (Fig. 4). In contrast, low MAF PIK3CA mutations were frequently found in ECs unrelated to OCCC and in eutopic endometrium. Although endometrial glands are considered monoclonal [10], our results suggest that sporadic PIK3CA mutations without clonal expansion occur in ECs and endometrial glands.

There are few PIK3CA mutations in HGSOC that are not associated with endometriosis [32], and no PIK3CA mutations were found in HGSOC tumors in our study. However, PIK3CA 
Table 2. PIK3CA mutations in eutopic endometrial glands.

\begin{tabular}{|c|c|c|c|c|c|c|c|c|c|c|c|}
\hline \multirow[t]{2}{*}{ Group } & \multicolumn{3}{|c|}{$\begin{array}{l}\text { Cases with mutations } \\
\text { (MAF } \geqq 15 \%) \text { in } \\
\text { endometrial glands }\end{array}$} & \multicolumn{3}{|c|}{$\begin{array}{l}\text { Number of hotspots with } \\
\text { mutations (MAF } \geqq 15 \% \text { ) }\end{array}$} & \multicolumn{2}{|c|}{$\begin{array}{l}\text { Mean MAF(\%) of } \\
\text { endometrial glands }\end{array}$} & \multicolumn{3}{|c|}{$\begin{array}{l}\text { Cases with multiple } \\
\text { hotspot mutations in } \\
\text { the same glands }\end{array}$} \\
\hline & & $\%$ & $P$ value & & $\%$ & $P$ value & $\%$ & $P$ value & & $\%$ & $P$ value \\
\hline OCCC without PIK3CA mutation & $11 / 12$ & 92 & 0.002 & $42 / 78$ & 54 & $<0.001$ & $32.4 \pm 35.4$ & $<0.001$ & $7 / 12$ & 58 & 0.003 \\
\hline HGSOC & $3 / 5$ & 60 & 0.31 & $8 / 45$ & 18 & $<0.001$ & $9.4 \pm 17.5$ & $<0.001$ & $1 / 5$ & 20 & 0.41 \\
\hline
\end{tabular}

OCCC ovarian clear cell carcinoma, HGSOC high-grade serous ovarian carcinoma, MAF mutant allele frequency.

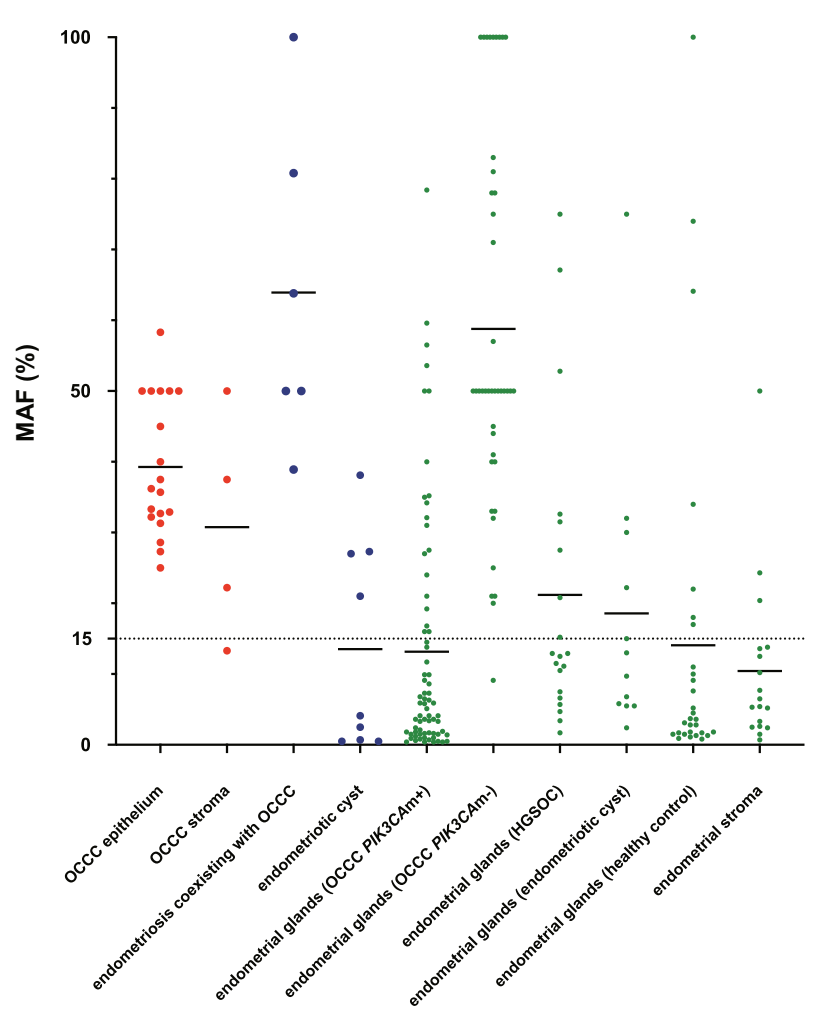

Fig. 4 Comparison of PIK3CA MAF in OCCC, endometriosis, and eutopic endometrium. Samples in which mutant alleles were detected are shown as dot plots for ten groups: OCCC (epithelial and stromal components of tumors sampled by laser-capture microdissection), endometriosis (comorbid endometriosis in OCCC and endometriotic cysts), and eutopic endometrium (endometrial glands of OCCC with PIK3CA mutation in the tumor, OCCC without PIK3CA mutation in the tumor, HGSOC, endometriotic cyst, healthy control, and endometrial stroma). The horizontal bar indicates the average. The dotted line indicates $\mathrm{MAF}=15 \%$. MAF mutant allele frequency, OCCC ovarian clear cell carcinoma, PIK3CAm PIK3CA mutation, HGSOC high-grade serous ovarian carcinoma.

mutations were found in the eutopic endometrial glands of HGSOC at a higher frequency than in the endometrial glands of controls (Fig. 2A, Table 2). In addition, analysis of the data by Wang et al. showed the presence of PIK3CA mutations in cell-free DNA of Pap smear and plasma in HGSOC cases (Supplementary Fig. S4). In a previous report on genome sequencing in normal uterine cervix, PIK3CA mutations were not detected at all [33]. The reason for the presence of PIK3CA mutations in Pap smear samples from ovarian cancer cases without PIK3CA mutations in the tumor may be that PIK3CA mutations are more likely to occur in the cervix in ovarian cancer cases. Alternatively, it is also possible that the PIK3CA mutation occurred in the eutopic endometrial glands, as we observed, and DNA or endometrial cells with PIK3CA mutation were introduced into the Pap smear samples. In ovarian cancer, cell-free DNA in plasma and tumor mutations do not always coincide, and intratumor heterogeneity has been commonly considered as a cause of this phenomenon [34]. Our data, however, indicate that other genetic variants of normal organ origin must be kept in mind. Individuals with a history of cancer, including OCCC, have a higher risk of developing a second cancer than individuals without a history of cancer [35-37]. This may be because the carcinogenic stress that caused the tumor in patients with carcinoma may also affect organs unrelated to the tumor, causing the accumulation of genetic variants in the other organs. Alternatively, considering the frequency with which humans develop cancer during their lifetime, many cancer-associated mutations that occur in normal tissues may be passenger mutations. The biological significance of cancer-associated mutations in normal tissues should be investigated in future studies.

Surprisingly, there were some cases in which the stromal component of OCCC had PIK3CA mutations (Fig. 3C). Initially, we thought that the stromal component would be composed of nontumor cells. We thought that it would be easy to distinguish the epithelial component from the stromal component in OCCC by histopathology (Fig. 3B) and that at the most, only a little epithelial component may be mixed in when the stromal component was sampled by LCM. Furthermore, PIK3CA mutations in nontumor cells, if any, were considered to be sporadic mutations without clonal expansion, unrelated to mutations in the tumor epithelium, and associated with low MAF. Therefore, the presence of PIK3CA mutations in the stromal component at a relatively high MAF, consistent with those in the epithelial component, was unexpected. This suggests that the apparently stromal component of OCCC also contains tumor cells. Previous studies showed that the side-population cells of endometrial cancer can differentiate into mesenchymal cells [38], and OCCC tumor cells may also exist as mesenchymal cells. Among the OCCC cases in this study, two had PIK3CA mutations with MAF $\geq 15 \%$ in the eutopic endometrial stroma, and both had the same hotspot mutation in the tumor (Fig. 2A). Moreover, in both cases, microdissection revealed identical hotspot mutations in all samples of the epithelial component of the tumor, and one showed homogeneous identical hotspot mutations not only in the epithelial component but also in the stromal component of the tumor (Fig. 3C). The eutopic endometrial stroma has been reported to contain few cancer-associated mutations [39]. However, our data suggest that low-frequency mutations in endometrial stromal cells contribute more to the true driver mutations of EAOC than high frequency, probably mostly passenger, cancer-associated mutations in endometrial glandular epithelial cells, and eutopic endometrial stromal cells may be the 
origin of EAOC. The fact that endometrial stromal cells have been used as material in many studies on endometriosis may also support our findings [40]. Our data, however, are based on a very limited number of cases and do not explain the process of transformation from stromal cells to epithelial cells, so we have only shown one possibility. Nevertheless, our results suggest that future studies of the eutopic endometrium for the purpose of searching for the origin of EAOC should examine not only the endometrial glandular epithelium but also the endometrial stroma.

In this study, hotspot mutations of PIK3CA were examined by ddPCR using FFPE samples because of the ease of sample collection. However, this method did not allow us to conclude whether the origin of OCCC was eutopic endometrium. We could not exclude the possibility that other cancerassociated mutations in the endometrial glands may be the direct origin of the tumor or that the endometrial glands and tumor may show clonality for other cancer-associated mutations. For future research purposes, it will be necessary to cryopreserve the removed specimens, including the normallooking uterus, when performing surgery for ovarian cancer. A large number of endometrial glands and stroma from the entire endometrium should then be collected by LCM and analyzed by whole exome or whole genome sequencing and compared with the data from tumor tissue. Further advances in sequencing technology are therefore needed for these studies.

In conclusion, here we found that PIK3CA mutations specific to endometrial glandular epithelium are frequently observed in eutopic endometrium with OCCC, and most of the mutations may not be related to the development of OCCC. Further comprehensive analysis is needed to clarify the significance of cancerassociated mutations in eutopic endometrium.

\section{DATA AVAILABILITY}

The datasets used and analyzed during the current study are available from the corresponding author on reasonable request.

\section{REFERENCES}

1. Nagase S, Ohta T, Takahashi F, Enomoto T. Annual report of the committee on gynecologic oncology, the Japan Society of Obstetrics and Gynecology: annual patients report for 2015 and annual treatment report for 2010. J Obstet Gynaecol Res. 2019;45:289-98.

2. Irving J, Clement P. Disease of the peritoneum. In: Kurman RJ, Ellenson LH, Ronnett BM, editors. Blaustein's pathology of the female genital tract. 7th ed. New York, NY: Springer; 2019. p. 816-20.

3. Vercellini $P$, Viganò $P$, Somigliana $E$, Fedele L. Endometriosis: pathogenesis and treatment. Nat Rev Endocrinol. 2014;10:261-75.

4. Lac V, Nazeran TM, Tessier-Cloutier B, Aguirre-Hernandez R, Albert A, Lum A, et al. Oncogenic mutations in histologically normal endometrium: the new normal? J Pathol. 2019;249:173-81.

5. Moore L, Leongamornlert D, Coorens THH, Sanders MA, Ellis P, Dentro SC, et al. The mutational landscape of normal human endometrial epithelium. Nature. 2020;580:640-6.

6. Anglesio MS, Papadopoulos N, Ayhan A, Nazeran TM, Noë M, Horlings HM, et al. Cancer-associated mutations in endometriosis without cancer. N Engl J Med. 2017;376:1835-48.

7. Suda K, Nakaoka H, Yoshihara K, Ishiguro T, Tamura R, Mori Y, et al. Clonal Expansion and diversification of cancer-associated mutations in endometriosis and normal endometrium. Cell Rep. 2018;24:1777-89.

8. Suda K, Cruz Diaz LA, Yoshihara K, Nakaoka H, Yachida N, Motoyama T, et al. Clonal lineage from normal endometrium to ovarian clear cell carcinoma through ovarian endometriosis. Cancer Sci. 2020;111:3000-9.

9. Murakami K, Kotani $\mathrm{Y}$, Nakai H, Matsumura N. Endometriosis-associated ovarian cancer: the origin and targeted therapy. Cancers. 2020;12:1676.

10. Tanaka M, Kyo S, Kanaya T, Yatabe N, Nakamura M, Maida Y, et al. Evidence of the monoclonal composition of human endometrial epithelial glands and mosaic pattern of clonal distribution in luminal epithelium. Am J Pathol. 2003;163:295-301.
11. Uchiyama $Y$, Nakashima $M$, Watanabe $S$, Miyajima $M$, Taguri $M$, Miyatake $S$, et al. Ultra-sensitive droplet digital PCR for detecting a low-prevalence somatic GNAQ mutation in Sturge-Weber syndrome. Sci Rep. 2016;6:22985.

12. Chui $\mathrm{MH}$, Shih IM. Oncogenic BRAF and KRAS mutations in endosalpingiosis. J Pathol. 2020;250:148-58.

13. Kang S, Bader AG, Vogt PK. Phosphatidylinositol 3-kinase mutations identified in human cancer are oncogenic. PNAS. 2004;102:802-7.

14. Fujiwara K, Shintani D, Nishikawa T. Clear-cell carcinoma of the ovary. Ann Oncol. 2016;27:50-52.

15. Chandler RL, Damrauer JS, Raab JR, Schisler JC, Wilkerson MD, Didion JP, et al. Coexistent ARID1A-PIK3CA mutations promote ovarian clear-cell tumorigenesis through pro-tumorigenic inflammatory cytokine signalling. Nat Commun. 2015;6:6118.

16. Kuo KT, Mao TL, Jones $S$, Veras $E$, Ayhan $A$, Wang $T L$, et al. Frequent activating mutations of PIK3CA in ovarian clear cell carcinoma. Am J Pathol. 2009;174:1597-601.

17. Yamamoto $S$, Tsuda $H$, Takano M, Tamai $S$, Matsubara O. PIK3CA mutations and loss of ARID1A protein expression are early events in the development of cystic ovarian clear cell adenocarcinoma. Virchows Arch. 2012;460:77-87.

18. Wang Y, Li L, Douville C, Cohen JD, Yen TT, Kinde I, et al. Evaluation of liquid from the Papanicolaou test and other liquid biopsies for the detection of endometrial and ovarian cancers. Sci Transl Med. 2018;10:eaap8793.

19. Yamamoto S, Tsuda H, Takano M, Iwaya K, Tamai S, Matsubara O. PIK3CA mutation is an early event in the development of endometriosis-associated ovarian clear cell adenocarcinoma. J Pathol. 2011;225:189-94.

20. Murakami R, Matsumura N, Brown JB, Higasa K, Tsutsumi T, Kamada M, et al. Exome sequencing landscape analysis in ovarian clear cell carcinoma shed light on key chromosomal regions and mutation gene networks. Am J Pathol. 2017;187:2246-58.

21. Shibuya $Y$, Tokunaga $H$, Saito $S$, Shimokawa $K$, Katsuoka F, Bin L, et al. Identification of somatic genetic alterations in ovarian clear cell carcinoma with next generation sequencing. Genes Chromosom Cancer. 2018;57:51-60.

22. Mina M, Raynaud F, Tavernari D, Battistello E, Sungalee S, Saghafinia S, et al. Conditional selection of genomic alterations dictates cancer evolution and oncogenic dependencies. Cancer Cell. 2017;32:155-68.

23. Sanchez-Vega F, Mina M, Armenia J, Chatila WK, Luna A, La KC, et al. Oncogenic signaling pathways in The Cancer Genome Atlas. Cell. 2018;173:321-37.

24. Yokoyama A, Kakiuchi N, Yoshizato T, Nannya Y, Suzuki H, Takeuchi Y, et al. Agerelated remodelling of oesophageal epithelia by mutated cancer drivers. Nature. 2019;565:312-7.

25. Martincorena I, Roshan A, Gerstung M, Ellis P, Loo PV, McLaren S, et al. High burden and pervasive positive selection of somatic mutations in normal human skin. Science. 2015;348:880-6.

26. Martincorena I, Fowler JC, Wabik A, Lawson ARJ, Abascal F, Hall MWJ, et al. Somatic mutant clones colonize the human esophagus with age. Science. 2018;362:911-7.

27. Yoshida K, Gowers KHC, Lee-Six H, Chandrasekharan DP, Coorens T, Maughan EF, et al. Tobacco smoking and somatic mutations in human bronchial epithelium. Nature. 2020;578:266-72.

28. Kakiuchi N, Yoshida $K$, Uchino $M$, Kihara $T$, Akaki $K$, Inoue $Y$, et al. Frequent mutations that converge on the NFKBIZ pathway in ulcerative colitis. Nature. 2020;577:260-5.

29. Lee-Six H, Olafsson S, Ellis P, Osborne RJ, Sanders MA, Moore L, et al. The landscape of somatic mutation in normal colorectal epithelial cells. Nature. 2019;574:532-7.

30. Anglesio MS, Bashashati A, Wang YK, Senz J, Ha G, Yang W, et al. Multifocal endometriotic lesions associated with cancer are clonal and carry a high mutation burden. J Pathol. 2015;236:201-9.

31. Parsons BL, McKim KL, Myers MB. Variation in organ-specific PIK3CA and KRAS mutant levels in normal human tissues correlates with mutation prevalence in corresponding carcinomas. Environ Mol Mutagen. 2017;58:466-76.

32. Bell D, Berchuck A, Birrer M, Chien J, Cramer DW, Dao F, et al. Integrated genomic analyses of ovarian carcinoma. Nature. 2011;474:609-15.

33. Yizhak K, Aguet F, Kim J, Hess JM, Kübler K, Grimsby J, et al. RNA sequence analysis reveals macroscopic somatic clonal expansion across normal tissues. Science. 2019;364:eaaw0726.

34. Asante DB, Calapre L, Ziman M, Meniawy TM, Gray ES. Liquid biopsy in ovarian cancer using circulating tumor DNA and cells: Ready for prime time? Cancer Lett. 2020;468:59-71.

35. Nguyen JMV, Vicus D, Nofech-Mozes S, Gien LT, Bernardini MQ, Rouzbahman M, et al. Risk of second malignancy in patients with ovarian clear cell carcinoma. Int J Gynecol Cancer. 2020. https://doi.org/10.1136/ijgc-2020-001946.

36. Wood ME, Vogel V, Ng A, Foxhall L, Goodwin P, Travis LB. Second malignant neoplasms: assessment and strategies for risk reduction. J Clin Oncol. 2012;30:3734-45. 
37. Bright CJ, Reulen RC, Winter DL, Stark DP, McCabe MG, Edgar AB, et al. Risk of subsequent primary neoplasms in survivors of adolescent and young adult cancer (Teenage and Young Adult Cancer Survivor Study): a population-based, cohort study. Lancet Oncol. 2019;20:531-45.

38. Kato K, Takao T, Kuboyama A, Tanaka Y, Ohgami T, Yamaguchi S, et al. Endometrial cancer side-population cells show prominent migration and have a potential to differentiate into the mesenchymal cell lineage. Am J Pathol. 2010;176:381-92.

39. Suda K, Nakaoka H, Yoshihara K, Ishiguro T, Adachi S, Kase H, et al. Different mutation profiles between epithelium and stroma in endometriosis and normal endometrium. Hum Reprod. 2019;34:1899-905.

40. Burney RO, Giudice LC. Pathogenesis and pathophysiology of endometriosis. Fertil Steril. 2012;98:511-9.

\section{ACKNOWLEDGEMENTS}

The authors thank Akiko Kyoda for FFPE sample preparation and toluidine blue staining.

\section{AUTHOR CONTRIBUTIONS}

$\mathrm{KM}$ and $\mathrm{NM}$ designed the study. $\mathrm{AK}, \mathrm{HN}$, and $\mathrm{YK}$ collected specimens for the study. KM and CM performed experiments. KS and HT performed data analysis. KM drafted the manuscript. KN and NM supervised the study. All authors were involved in writing the paper and had final approval of the submitted and published versions.

\section{FUNDING}

This study was supported in part by Japan Society for the Promotion of Science (JSPS) KAKENHI grant number $18 \mathrm{H} 02947$ (Grant-in-Aid for Scientific Research B for Noriomi Matsumura) and 20K21665 (Grant-in-Aid for Challenging Exploratory Research for Noriomi Matsumura).

\section{COMPETING INTERESTS}

The authors declare no competing interests.

\section{ETHICS APPROVAL}

This study was conducted with the approval of the Institutional Review Board of Kindai University Faculty of Medicine (27-182). Patients in this study were given an appropriate opportunity to refuse to participate in the study by opt out on the website of Kindai University Faculty of Medicine (https://www.kindai.ac.jp/medicine/) This study was performed in accordance with the Declaration of Helsinki.

\section{ADDITIONAL INFORMATION}

Supplementary information The online version contains supplementary material available at https://doi.org/10.1038/s41379-021-00861-3.

Correspondence and requests for materials should be addressed to N.M.

Reprints and permission information is available at http://www.nature.com/ reprints

Publisher's note Springer Nature remains neutral with regard to jurisdictional claims in published maps and institutional affiliations.

\begin{abstract}
cC) Open Access This article is licensed under a Creative Commons Attribution 4.0 International License, which permits use, sharing, adaptation, distribution and reproduction in any medium or format, as long as you give appropriate credit to the original author(s) and the source, provide a link to the Creative Commons license, and indicate if changes were made. The images or other third party material in this article are included in the article's Creative Commons license, unless indicated otherwise in a credit line to the material. If material is not included in the article's Creative Commons license and your intended use is not permitted by statutory regulation or exceeds the permitted use, you will need to obtain permission directly from the copyright holder. To view a copy of this license, visit http://creativecommons. org/licenses/by/4.0/.
\end{abstract}

(c) The Author(s) 2021 